\title{
Necessidades de Cuidados e Recurso aos Serviços na Demência: Avaliação Inicial da Coorte Portuguesa no Estudo Actifcare
}

\author{
Needs for Care and Service Use in Dementia: Baseline \\ Assessment of Portuguese Participants in the Actifcare \\ Cohort Study
}

\author{
Manuel GONÇALVES-PEREIRA ${ }^{1}{ }^{1}$, Maria J. MARQUES ${ }^{1}$, Conceição BALSINHA ${ }^{1,2}$, Alexandra FERNANDES ${ }^{3}$, \\ Ana Sá MACHADO ${ }^{1}$, Ana VERDELHO ${ }^{4}$, Bernardo BARAHONA-CORREAA ${ }^{5,6}$, Helena BÁRRIOS ${ }^{5}$, João GUIMARÃES ${ }^{5}$, \\ Joana GRAVE ${ }^{5}$, Luísa ALVES ${ }^{1,6}$, Manuel Caldas de ALMEIDA ${ }^{5}$, Teresa Alves REIS ${ }^{1}$, Martin ORRELL ${ }^{7}$, Bob WOODS ${ }^{8}$, \\ Marjolein de VUGT $^{9}$, Frans VERHEY ${ }^{9}$, em representação do ACTIFCARE CONSORTIUM \\ Acta Med Port 2019 May;32(5):355-367 • https://doi.org/10.20344/amp.11136
}

\section{RESUMO}

Introdução: As pessoas com demência e os seus familiares deveriam ter acesso atempado a cuidados formais na comunidade (centros de dia, apoio domiciliário). O projecto EU-Actifcare investigou o acesso/utilização destes serviços em países europeus. Descrevemos a implementação do estudo de coorte e a avaliação inicial em Portugal, com foco nas necessidades de cuidados e recurso aos serviços.

Material e Métodos: Selecionámos uma amostra de conveniência de 66 pessoas com diagnóstico de demência ligeira a moderada (residindo na comunidade sem cuidados formais relevantes) e respetivos familiares-cuidadores. A avaliação (clínico-funcional e social) incluiu os instrumentos Camberwell Assessment of Need for the Elderly e Resource Utilization in Dementia.

Resultados: Identificámos necessidades não-cobertas dos doentes (média 1,1; DP $=1,7)$, principalmente de companhia (23\% dos casos), sofrimento psicológico (20\%) e atividades diárias (14\%). Os familiares-cuidadores dedicavam 150 minutos/dia (mediana) à prestação de cuidados e $44 \%$ apresentavam necessidades não-cobertas de sofrimento psicológico. Quando havia problemas de acesso/utilização dos serviços de saúde e sociais na comunidade, estes estavam frequentemente relacionados com recusa ou desconhecimento de utentes/familiares.

Discussão: A seleção dos participantes não foi fácil, pela especificidade dos critérios adotados. Não almejando representatividade nacional, recrutámos uma amostra típica de pessoas em estádios ligeiros a moderados de demência, em serviços e regiões diferentes. Nalguns casos, encontrámos necessidades não-cobertas e repercussões familiares que já justificariam respostas de serviços na comunidade, não fossem os problemas de acesso/utilização.

Conclusão: Na área das demências, existem dificuldades no acesso atempado e utilização efectiva de cuidados formais, coexistindo com uma cobertura menor de necessidades específicas.

Palavras-chave: Acesso aos Serviços de Saúde; Cuidadores; Demência; Determinação de Necessidades de Cuidados de Saúde; Portugal

\section{ABSTRACT}

Introduction: People with dementia and their relatives should have timely access to formal care in the community. The EU-Actifcare project analysed access to and use of formal services, as related to unmet needs for care. We describe the cohort study implementation and baseline results in Portugal, with a focus on needs for care and service use assessments.

Material and Methods: Our convenience sample consisted of 66 dyads of community-dwelling people with mild to moderate dementia and no significant use of formal services, and their informal carers. Measures included the Camberwell Assessment of Need for the Elderly and Resources Utilization in Dementia.

Results: People with dementia had unmet needs (mean 1.1; SD 1.7), mainly regarding company (23\%), psychological distress (20\%), and daily activities (14\%). Family caregivers spent 150 minutes/day (median) providing support, and $44 \%$ had psychological distress unmet needs. Problems with access to or use of formal services, when present, were frequently due to attitudes or lack of knowledge of any or both members of the dyad.

Discussion: The recruitment process was challenging, since the inclusion criteria were restrictive. Not claiming generalizability, we recruited a typical sample of Portuguese people with mild to moderate dementia and no significant formal community support. Levels and type of unmet needs found in some participants would call for formal support, were it not for problems regarding access or use.

1. Centro de Estudos de Doenças Crónicas - CEDOC. Faculdade de Ciências Médicas / NOVA Medical School. Universidade NOVA de Lisboa. Lisboa. Portugal.

2. Unidade de Saúde Familiar Marginal. Agrupamento dos Centros de Saúde de Cascais. Cascais. Portugal.

3. Unidade de Saúde Familiar Fernão Ferro Mais. Agrupamento dos Centros de Saúde de Almada-Seixal. Seixal. Portugal

4. Instituto de Saúde Ambiental. Faculdade de Medicina. Centro Hospitalar e Universitário de Lisboa. Lisboa. Portugal.

5. Faculdade de Ciências Médicas / NOVA Medical School. Universidade NOVA de Lisboa. Lisboa. Portugal.

6. Hospital de Egas Moniz. Centro Hospitalar de Lisboa Ocidental. Lisboa. Portugal.

7. Nottingham Institute of Mental Health. Nothingham. United Kingdom.

8. Bangor University. Bangor. United Kingdom.

9. Maastricht University. Maastricht. The Netherlands.

$\triangle$ Autor correspondente: Manuel Gonçalves-Pereira. gpereira@nms.unl.pt

Recebido: 01 de agosto de 2018 - Aceite: 10 de dezembro de 2018 | Copyright @ Ordem dos Médicos 2019 
Conclusion: There are difficulties regarding timely access and effective use of formal care in dementia, along with relevant unmet needs.

Keywords: Caregivers; Dementia; Health Services Accessibility; Needs Assessment; Portugal

\section{INTRODUÇÃO}

Num contexto de envelhecimento global, o número de pessoas com demência tem aumentado substancialmente, podendo atingir 131,5 milhões em 2050 e uma 'carga de doença' (burden of disease) enorme. ${ }^{1}$ Em Portugal, foram estimados 160287 casos acima dos 60 anos em 2013, ${ }^{2}$ números que serão maiores considerando a prevalência recentemente determinada em Portugal pelo método do 10/66-Dementia Research Group (9,23\%; IC 95\% 7,80 $10,90) .^{3}$

As demências são entidades clínicas complexas, envolvendo múltiplas necessidades bio-psico-sociais. ${ }^{4,5}$ Este conceito de 'necessidades de saúde' implica respostas desde a promoção da saúde aos vários níveis de prevenção. ${ }^{6}$ Em investigação de serviços, uma 'necessidade (de cuidados)' é um problema para o qual existe uma intervenção 'adequada', i.e., uma resposta baseada na evidência com potencial para cobrir (satisfazer), total ou parcialmente, essa 'necessidade'.,5 Internacionalmente, a avaliação de necessidades nas demências tem assumido grande relevância. ${ }^{7}$ Também em Portugal foram documentadas necessidades, nem sempre atendidas, em diversos quadros psicogeriátricos. ${ }^{8-10}$ Por outro lado, a maioria destas pessoas tem apoio de familiares, os quais apresentam necessidades próprias: ${ }^{11}$ muitos cuidadores informais apresentam grande sobrecarga (objetiva e subjetiva) ${ }^{12}$ e sofrimento psíquico, ${ }^{12,13}$ como documentado igualmente em Portugal. ${ }^{14,15}$

Neste cenário, as respostas dos serviços são insuficientes. ${ }^{16,17}$ Um diagnóstico mais precoce melhoraria os cuidados de muitos doentes e familiares..$^{18}$ Apesar das tentativas para implementar um diagnóstico atempado, persistem barreiras importantes, frequentemente por normalização dos sintomas ou falta de informação. 19,20 Também o estigma atrasa a procura do diagnóstico ou o acesso aos cuidados, com eventual exaustão familiar e institucionalizações prematuras. ${ }^{18,21}$ Acrescem falhas na referenciação para os cuidados secundários (Neurologia, Psiquiatria) e na articulação inter/intra-serviços. ${ }^{16,22,23}$

A investigação de serviços tem-se concentrado nas fases precoces ou avançadas das demências, e menos nas intermédias, em que são cruciais o acesso atempado e o uso regular dos serviços na comunidade (e.g. apoio domiciliário). Quando os cuidados informais deixam de ser suficientes, estes cuidados formais podem cobrir necessidades decorrentes da evolução clínica, com melhoria da qualidade de vida de doentes e familiares, redução da sobrecarga familiar e dos custos associados, ou adiando institucionalizações. ${ }^{24,25}$ Contudo, registam-se problemas no acesso e utilização continuada dos cuidados formais. ${ }^{20,26}$

A utilização dos serviços de saúde é analisável pelo Modelo de Andersen, ${ }^{27}$ recentemente revisto. ${ }^{28,29} \mathrm{O}$ acesso aos cuidados formais depende da interação entre determinan- tes individuais e contextuais. Os primeiros incluem fatores predisponentes para a utilização (e.g. sociodemográficos, crenças de saúde), facilitadores (e.g. recursos financeiros, seguros de saúde, listas de espera) e relacionados com necessidades de doentes e famílias (subjetivas ou diagnosticadas por outrem). O nível contextual desdobra-se, igualmente, em fatores predisponentes (e.g. sociodemográficos, culturais), facilitadores (e.g. despesas com saúde, serviços e equipamentos, políticas de saúde) e alusivos às necessidades ambientais/indicadores populacionais (e.g. esperança de vida, mortalidade, morbilidade, níveis de incapacidade). A dinâmica inter-fatorial é especialmente complexa em condições como a demência, pois as necessidades alteram-se progressivamente.

Tal como outros estudos, ${ }^{30}$ o projecto Actifcare (ACcess to TImely Formal Care) da Programação Conjunta da União Europeia-Investigação em Doenças Neurodegenerativas/ JPND (2014 - 2017) inspirou-se no modelo de Andersen. O projeto incidiu na fase intermédia das demências (na transição dos cuidados informais, quando estes se tornam insuficientes, para combinações entre estes e os cuidados formais na comunidade e.g., apoio domiciliário, centros de dia). ${ }^{31}$ Focámo-nos no acesso e utilização destes serviços em oito países Europeus (Alemanha, Holanda, Irlanda, Itália, Noruega, Portugal, Reino Unido e Suécia), avaliando necessidades não-cobertas e qualidade de vida em doentes e seus familiares. Interessaram-nos, também, a disponibilidade, acessibilidade e utilização dos serviços nos vários países. ${ }^{31}$ Este projeto global incluiu diversos componentes (work packages-WPs): WP2 (revisões da literatura; estudo qualitativo das experiências de doentes, cuidadores informais, profissionais; perspetivas político-administrativas), WP3 (estudo longitudinal sobre acesso/utilização dos cuidados formais), WP4 (análise custo-efetividade, baseada no WP3), WP5 (identificação de boas práticas e recomendações). ${ }^{31}$

O estudo longitudinal (WP3) analisou, ao longo de um ano nos oito países, os fatores predisponentes, facilitadores e barreiras no recurso aos cuidados formais, a par da qualidade de vida e necessidades dos participantes. ${ }^{31}$ No presente artigo, incidimos na avaliação inicial (baseline) da coorte portuguesa deste estudo, com enfoque nas necessidades de cuidados e no acesso/utilização dos serviços.

\section{MATERIAL E MÉTODOS}

Desenho

O protocolo geral do projeto EU-Actifcare está disponível (www.actifcare.eu), incluindo a metodologia do estudo de coorte (WP3) ${ }^{31}$ : estudo longitudinal, observacional, com seguimento (follow-up) aos seis e doze meses. Descrevemos aqui apenas a avaliação inicial da amostra, realçando particularidades da implementação em Portugal. 


\section{Participantes}

Selecionámos díades de pessoas com demência e respectivos cuidadores informais principais.

Critérios de inclusão: diagnóstico DSM-IV-TR de demência, segundo o médico responsável;32 estádio ligeiro a moderado (pontuação 1 ou 2 na escala Clinical Dementia Rating-CDR); ${ }^{33}$ pontuação < 25 na escala Mini Mental State Examination (MMSE) ${ }^{34}$; existência de cuidador não remunerado (informal) em contacto regular (mínimo: uma vez por semana); avaliação subjetiva do clínico responsável dando como provável o início da necessidade de cuidados formais no ano subsequente (para que o follow-up permitisse estudar um número apreciável de transições para cuidados formais). Os 'cuidados formais na comunidade' foram definidos operacionalmente como serviços de apoio domiciliário ou 'de dia' (e.g. centros), ou outro apoio regular (no mínimo, semanal) envolvendo técnicos ou cuidadores remunerados.

Critérios de exclusão (doentes): demência alcoólica, doença de Huntington (o diagnóstico do subtipo de demência, quando existente, era reportado pelo clínico responsável); cuidados formais relevantes devido a demência, no momento ou nos seis meses anteriores (comunitários ou institucionais e.g., para alívio familiar).

Critérios de exclusão (doentes/cuidadores): limitações graves de linguagem, audição ou literacia; comorbilidade relevante (e.g. doença grave, mental ou outra, perturbação do desenvolvimento).

O protocolo EU-Actifcare previa o recrutamento de, pelo menos, 50 díades por país, para perfazer 400. Não sendo possível assegurar representatividade nacional, as nossas amostras de conveniência deveriam evitar reproduzir situações atípicas a nível nacional. Em Portugal, antecipando dificuldades no recrutamento pelo carácter restritivo dos critérios (acima), articulámos com cuidados primários, consultas de Neurologia e Psiquiatria, serviços privados e associações sem fins lucrativos (e.g. Alzheimer Portugal) em diversas regiões (Lisboa, margem sul do Tejo, Cascais, Évora, Mora, Gouveia).

\section{Medidas}

Variáveis em estudo e instrumentos de avaliação constam da Tabela $1 .{ }^{31}$ Muitos instrumentos tinham versões portuguesas validadas. ${ }^{31}$ Não reportamos aqui a validação preliminar dos restantes, nem os dados sobre qualidade de vida, relação interpessoal na díade, suporte social e locus de controlo (Tabela 1).

\section{Avaliação das pessoas com demência}

Neste artigo, focado na avaliação de necessidades e na descrição da utilização dos serviços, reportamos sobretudo dados de duas entrevistas: Camberwell Assessment of Need for the Elderly (CANE) ${ }^{4,5}$ e Resources Utilization in Dementia (RUD). ${ }^{35}$ A entrevista CANE avalia a existência de necessidades (não) cobertas de cuidados, numa abordagem 'centrada na pessoa': considera, quanto ao doente, 24 domínios (biológicos, psicológicos ou sociais) e permite comparar as perspetivas dos doentes, dos seus cuidadores informais e dos profissionais envolvidos (sendo as três consideradas numa quarta pontuação, integrada, do investigador/avaliador). Em cada domínio (item) uma necessidade pode inexistir, estar coberta ou não coberta. A pontuação total resulta da soma dos itens em que se registem necessidades. $^{8} \mathrm{O}$ instrumento apresenta validade e fiabilidade (teste-reteste, inter-observadores) robustas, ${ }^{5}$ com propriedades psicométricas confirmadas internacionalmente..$^{7,36}$ Em Portugal, foi traduzida, ${ }^{8}$ validada ${ }^{9}$ e usada em investigação de serviços. ${ }^{10,14}$ Neste estudo, considerámos as perspetivas dos doentes, cuidadores informais e avaliadores.

A entrevista RUD, traduzida para 58 línguas, mede a utilização dos recursos formais e informais na demência. ${ }^{35}$ Quanto aos últimos, obtivemos informação sobre o tempo despendido pelo familiar-cuidador na prestação de cuidados/supervisão. O instrumento apresenta boas propriedades psicométricas (validade facial, fiabilidade). ${ }^{35} \mathrm{~A}$ avaliação do acesso/utilização dos serviços formais foi complementada com uma lista de verificação ad hoc. ${ }^{31}$ Procedemos ainda à caracterização clínico-funcional dos doentes: gravidade da demência, ${ }^{33}$ desempenho cognitivo, ${ }^{34}$ sintomas neuropsiquiátricos ${ }^{37}$, comorbilidades, ${ }^{38}$ e desempenho funcional ${ }^{39}$ (Tabela 1).

\section{Avaliação dos cuidadores informais}

Avaliámos necessidades de informação sobre demência e relacionadas com sofrimento psicológico (dois itens específicos dos cuidadores na entrevista CANE), ${ }^{4,5}$ e utilização de recursos (RUD). ${ }^{35}$ Aferimos ainda (Tabela 1): sintomatologia ango-depressiva, ${ }^{40}$ sobrecarga familiar pela doença, ${ }^{41}$ tempo de perseverança ${ }^{42}$ e sentido de coerência. ${ }^{43}$ A Hospital Anxiety and Depression Scale (HADS) quantifica sintomas de depressão e/ou ansiedade. Estão validados internacionalmente pontos de corte nas subescalas 'depressão' e 'ansiedade': $\geq 11$ (identificação de casos prováveis) e $\geq 8$ (casos possíveis). ${ }^{40,44} \mathrm{Em}$ Portugal, estes limiares não foram validados em sentido estrito, ${ }^{45}$ sendo utilizados condicionalmente.

\section{Procedimentos}

\section{Treino dos entrevistadores}

Selecionámos dez entrevistadores/facilitadores (psicólogos clínicos) e conduzimos quatro sessões de treino para administração e cotação dos instrumentos (vídeos, role-play), focadas na entrevista CANE e na concordância inter-observadores. Promovemos esclarecimentos nos pontos de recrutamento.

\section{Estudo-piloto}

O estudo-piloto $(n=5)$ realizou-se em Lisboa, permitindo aperfeiçoar as versões portuguesas dos instrumentos e harmonizar procedimentos.

\section{Trabalho de campo}

As entrevistas foram agendadas no domicílio ou noutro local conveniente, assegurando privacidade e conforto. 
Tabela 1 - Instrumentos utilizados no estudo de coorte

\begin{tabular}{|c|c|c|c|}
\hline Instrumento & Variável & Respondente $^{a}$ & $\begin{array}{l}\text { Autor (ano) / Versão Portuguesa (ano) e } \\
\text { referência da validação nacional }\end{array}$ \\
\hline \multicolumn{4}{|c|}{ Caracterização sociodemográfica / clínica das pessoas com demência } \\
\hline Questionário sociodemográfico & Dados sociodemográficos & 1,2 & Grupo de Trabalho Actifcare em Portugal (2015) \\
\hline Clinical Dementia Rating (CDR) & Gravidade da demência & 1,2 & $\begin{array}{l}\text { Morris (1993) / Grupo de Estudos de Envelhecimento } \\
\text { Cerebral e Demência (2008) }\end{array}$ \\
\hline Mini Mental State Examination (MMSE) & Cognição & 1 & $\begin{array}{l}\text { Folstein et al (1975) / Guerreiro et al (1994); versão } \\
\text { portuguesa obtida através da Psychological } \\
\text { Assessment Resources, via MAPI Research Trust - } \\
\text { PROQOLID (2014) }\end{array}$ \\
\hline $\begin{array}{l}\text { Neuropsychiatric Inventory Questionnaire } \\
\text { (NPI-Q) }\end{array}$ & Sintomas neuropsiquiátricos & 2 & $\begin{array}{l}\text { Kaufer et al (2000) / Adaptado de Grupo de Estudos } \\
\text { de Envelhecimento Cerebral e Demência (2008); } \\
\text { autorização obtida através do website } \\
\text { http://www.npitest.net/ (2014) }\end{array}$ \\
\hline
\end{tabular}

\begin{tabular}{|c|c|c|c|}
\hline Índice de Charlson & Comorbilidade & 4 & $\begin{array}{l}\text { Charlson et al (1994) / Grupo de Trabalho Actifcare em } \\
\text { Portugal (2015) }\end{array}$ \\
\hline $\begin{array}{l}\text { Lawton Instrumental Activities of Daily } \\
\text { Living (IADL) }\end{array}$ & $\begin{array}{l}\text { Atividades instrumentais de vida } \\
\text { diária }\end{array}$ & 2 & Lawton \& Brody (1969) / Araújo et al (2008) \\
\hline Physical Self-Maintenance Scale (PSMS) & Atividades de vida diária & 2 & Lawton \& Brody (1969) / Araújo et al (2008) \\
\hline
\end{tabular}

\begin{tabular}{llcl}
\hline Acesso e utilização de serviços & & \\
\hline $\begin{array}{l}\text { Lista de verificação (checklist) de } \\
\text { utilização de serviços }\end{array}$ & $\begin{array}{l}\text { Acesso e razões para a (não) } \\
\text { utilização de serviços }\end{array}$ & 1,2 & Grupo de Trabalho Actifcare em Portugal (2015) \\
$\begin{array}{l}\text { Resources Utilization in Dementia (RUD) } \\
5.0^{\text {b }}\end{array}$ & Utilização de serviços & 1,2 & $\begin{array}{l}\text { Wimo et al (2013)/Versão portuguesa obtida através } \\
\text { do website http://rudinstrument.com (2015) }\end{array}$ \\
\hline
\end{tabular}

\begin{tabular}{|c|c|c|c|}
\hline \multicolumn{4}{|c|}{ Necessidades de cuidados, qualidade de vida, qualidade da relação } \\
\hline $\begin{array}{l}\text { Camberwell Assessment of Need for the } \\
\text { Elderly (CANE) }\end{array}$ & Necessidades de cuidados & $1,2,4$ & $\begin{array}{l}\text { Reynolds et al (2000) / Gonçalves-Pereira et al (2007); } \\
\text { Fernandes et al (2009) }\end{array}$ \\
\hline EQ-5D-5L / EQ-VAS & $\begin{array}{l}\text { Qualidade de vida relacionada } \\
\text { com a saúde }\end{array}$ & $1,2,3$ & Brooks (1996) / Ferreira et al (2013) \\
\hline DEMQOL-U / DEMQOL- Proxy-U & $\begin{array}{l}\text { Qualidade de vida da pessoa com } \\
\text { demência }\end{array}$ & 1,3 & $\begin{array}{l}\text { Mulhern et al (2013) / Grupo de Trabalho Actifcare em } \\
\text { Portugal (2015) }\end{array}$ \\
\hline $\begin{array}{l}\text { Quality of Life-Alzheimer's Disease } \\
\text { (QOL-AD) }\end{array}$ & $\begin{array}{l}\text { Qualidade de vida da pessoa com } \\
\text { demência }\end{array}$ & 1,3 & Logsdon et al (1999) / Bárrios et al (2013) \\
\hline CarerQoL-7D / CarerQoL-VAS & Qualidade de vida do cuidador & 2 & $\begin{array}{l}\text { Brouwer et al (2006) / Grupo de Trabalho Actifcare em } \\
\text { Portugal (2015) }\end{array}$ \\
\hline $\begin{array}{l}\text { ICEpop CAPability Measure for Older } \\
\text { People (ICECAP-O) }\end{array}$ & $\begin{array}{l}\text { Capacidade/qualidade de vida } \\
\text { do cuidador e da pessoa com } \\
\text { demência }\end{array}$ & $1,2,3$ & $\begin{array}{l}\text { Coast et al (2008) / Grupo de Trabalho Actifcare em } \\
\text { Portugal (2015) }\end{array}$ \\
\hline Positive Affect Index (PAI) & $\begin{array}{l}\text { Qualidade da relação do cuidador } \\
\text { e da pessoa com demência }\end{array}$ & 1,2 & $\begin{array}{l}\text { Bengtson \& Schrader (1982) / Grupo de Trabalho } \\
\text { Actifcare em Portugal (2015) }\end{array}$ \\
\hline \multicolumn{4}{|c|}{ Sintomatologia ango-depressiva, sobrecarga e suporte social do cuidador informal } \\
\hline $\begin{array}{l}\text { Hospital Anxiety and Depression Scale } \\
\text { (HADS) }\end{array}$ & Ansiedade e depressão & 2 & $\begin{array}{l}\text { Zigmond \& Snaith (1983) / Pais-Ribeiro et al (2007); } \\
\text { versão portuguesa obtida da GL Assessment, via MAPI } \\
\text { Research Trust - PROQOLID (2014) }\end{array}$ \\
\hline Relative Stress Scale (RSS) & Sobrecarga familiar pela doença & 2 & $\begin{array}{l}\text { Greene et al (1982) / Grupo de Trabalho Actifcare em } \\
\text { Portugal (2015) }\end{array}$ \\
\hline Lubben Social Network Scale (LSNS-6) & Suporte social 'percebido' & 2 & Lubben, 1988 / Ribeiro et al (2012) \\
\hline Tempo de perseverança & Questão únicad $^{d}$ & 2 & $\begin{array}{l}\text { Kraijo et al (2014) / Grupo de Trabalho Actifcare em } \\
\text { Portugal (2015) }\end{array}$ \\
\hline \multicolumn{4}{|c|}{ Locus de controlo, sentido de coerência do cuidador } \\
\hline Locus of Control Behaviour Scale (LOC) ${ }^{c}$ & Locus de controlo & 2 & $\begin{array}{l}\text { Craig et al (1984) / Grupo de Trabalho Actifcare em } \\
\text { Portugal (2015) }\end{array}$ \\
\hline $\begin{array}{l}\text { Orientation to Life Questionnaire } \\
\text { (SOC-13) }\end{array}$ & Sentido de coerência & 2 & Antonovsky (1987) / Saboga-Nunes (1999) \\
\hline
\end{tabular}

a Pessoa com demência (1); cuidador informal/familiar (2); cuidador informal (como proxy, dando a sua opinião sobre a situação da pessoa com demência ou assinalando a resposta que considera que o familiar com demência daria) (3); investigador/entrevistador (4); ' ${ }^{\circ}$ Aplicação de itens selecionados; ${ }^{\circ}$ Instrumentos usados apenas na primeira avaliação; ‘ 'Se a situação de cuidados se mantiver tal como está, quanto tempo será capaz de continuar a prestar cuidados?' 
Eram conduzidas separadamente com o doente e o familiar-cuidador (havendo momentos conjuntos, quando adequado, e sendo repartíveis em dois momentos para reduzir a sobrecarga avaliativa).

\section{Controlo de qualidade}

A recolha de dados respeitou o protocolo europeu, com monitorização pela coordenação do trabalho de campo nacional (MJM, MGP) e controlo da qualidade dos dados inseridos no sistema MACRO (coordenação WP3/Universidade de Bangor). Um segundo nível de controlo de qualidade (europeu) envolveu a verificação cruzada, aleatória, de dados inseridos: cada centro verificou dados de outro, até consenso final.

\section{Análise estatística}

Realizámos uma análise exploratória das variáveis de interesse, seguida de um estudo inferencial utilizando testes não paramétricos (Kruskall-Wallis, Mann-Whitney, qui-quadrado) ou paramétricos, consoante as condições de aplicabilidade. Analisando a concordância entre as perspetivas das pessoas com demência e dos seus cuidadores informais quanto às necessidades não-cobertas, calculámos os coeficientes Kappa $(-1$ a +1$)$ para cada item da entrevista CANE (a perspetiva dos entrevistadores, integrando as duas anteriores, não foi aqui considerada). Dependendo do valor de Kappa, a concordância foi considerada fraca ( $\mathrm{K}<0,20)$; ligeira $(0,21-0,40)$; moderada $(0,41-0,60)$; boa $(0,61-0,80)$ ou muito boa $(K>0,81) .{ }^{46}$ Considerámos um nível de significância $\alpha=0,05$. Usámos o Statistical Package for the Social Sciences/SPSS, v23.0 para Windows.

\section{Aspetos éticos e formais}

O consentimento informado foi assinado pelas pessoas com demência e pelos familiares-cuidadores. $O$ estudo foi autorizado, em Portugal, por Comissões de Ética (NMS/Faculdade de Ciências Médicas; Centro Hospitalar de Lisboa Ocidental; ARS Lisboa e Vale do Tejo; ARS Alentejo) e pela Comissão Nacional de Proteção de Dados. Formalizámos acordos com a Alzheimer Portugal, Santa Casa da Misericórdia de Mora, Hospital do Mar-Luz Saúde e Associação de Beneficência Popular de Gouveia.

\section{RESULTADOS}

\section{Caracterização demográfica e clínica dos participantes}

Avaliámos 66 díades de pessoas com demência e familiares-cuidadores, após exclusão de 17 que não cumpriam critérios para recrutamento numa análise mais minuciosa. Os participantes viviam em regiões diferentes (18 municípios), provindo de consultas de Medicina Geral e Familiar, Neurologia e Psiquiatria (principalmente em instituições públicas, mas também privadas) ou entidades sem fins lucrativos (Fig. 1).

Resumimos as características dos participantes portugueses na Tabela 2, contextualizando-as na amostra EU-Actifcare. ${ }^{47} \mathrm{~A}$ idade dos doentes variou entre 57 e 91 anos (média 77,3). Quanto à escolaridade, 30,3\% não a tinham, $45,5 \%$ apresentavam o ensino básico, $12,1 \%$ o secundário e $12,1 \%$ um grau superior. Quase 2/3 eram mulheres. O diagnóstico mais comum foi doença de Alzheimer $(37,9 \%)$ e a maioria $(89,4 \%)$ classificada como 'demência ligeira' (CDR 1). Encontrámos comorbilidade em 41 casos $(62,1 \%)$, e.g. diabetes: 20 (30,3\%); úlcera péptica: $6(9,1 \%)$; coronariopatia: 5 (7,6\%); vasculopatia periférica: 5 (7,6\%).

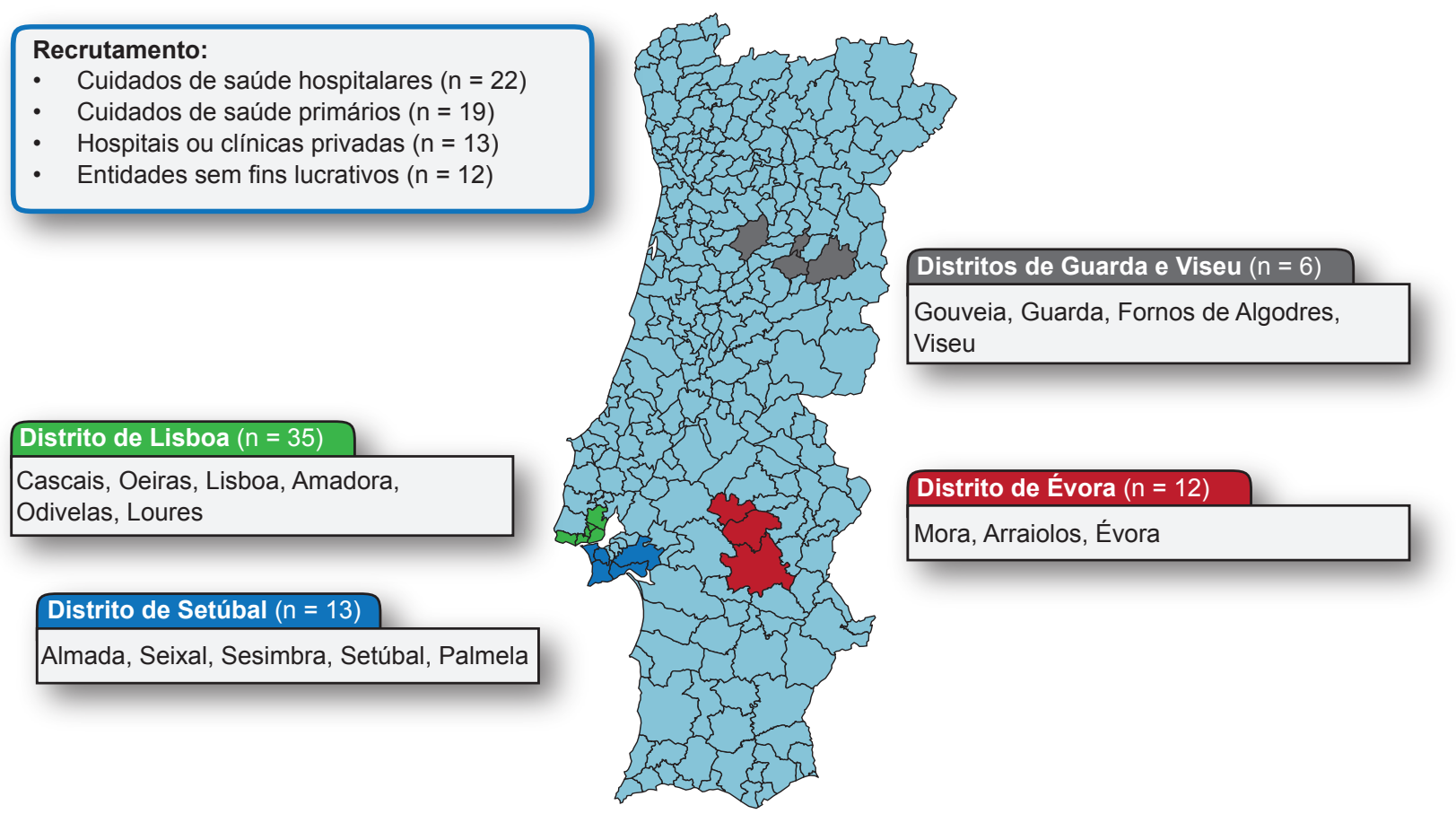

Figura 1 - Recrutamento e distribuição regional dos participantes 
Tabela 2 - Características sociodemográficas e clínicas das pessoas com demência e dos seus familiares/cuidadores informais: participantes portugueses e não portugueses EU-Actifcare

\begin{tabular}{|c|c|c|c|}
\hline Pessoas com demência & $\begin{array}{l}\text { Participantes portugueses } \\
\qquad(n=66)\end{array}$ & $\begin{array}{l}\text { Participantes não portugueses } \\
\qquad(\mathrm{n}=385)^{\mathrm{a}}\end{array}$ & Valor $p$ \\
\hline Idade, anos, média (DP) & $77,3(6,2)$ & $77,8(8,1)$ & 0,516 \\
\hline Sexo, feminino, n (\%) & $41(62,1)$ & $205(53,2)$ & 0,228 \\
\hline Educação, anos, média (DP) & $6,4(6,1)$ & $10,4(3,8)$ & 0,001 \\
\hline Tipos de demência, n (\%) & & & 0,169 \\
\hline Alzheimer & $25(37,9)$ & $193(50,4)$ & \\
\hline Vascular & $8(12,1)$ & $44(11,5)$ & \\
\hline Misto & $7(10,6)$ & $49(12,8)$ & \\
\hline Doença de Corpos de Lewy & $2(3,0)$ & $4(1,0)$ & \\
\hline Outro & $4(6,1)$ & $23(6,0)$ & \\
\hline Não especificado & $20(30,3)$ & $72(18,7)$ & \\
\hline CDR categorias 1 / 2, n (\%) & $59(89,4) / 7(10,6)$ & $\begin{array}{c}295(76,6) / 80(20,8) / 10(2,6) \\
\text { sem informação }\end{array}$ & 0,052 \\
\hline MMSE, média (DP) & $17,8(4,8)$ & $19,2(4,9)$ & 0,033 \\
\hline NPI valor total, média (DP) & $6,8(5,5)$ & $7,9(5,5)$ & 0,139 \\
\hline Comorbilidade (Charlson), média (DP) & $1,8(0,9)$ & $1,6(0,7)$ & 0,110 \\
\hline Ausência de comorbilidade, n (\%) & $25(37,9)$ & $194(51,1)$ & \\
\hline Comorbilidade baixa, $\mathrm{n}(\%)^{\mathrm{b}}$ & $31(47,0)$ & $131(34,5)$ & \\
\hline Comorbilidade elevada, $\mathrm{n}(\%)^{\mathrm{b}}$ & $10(15,2)$ & $55(14,5)$ & \\
\hline Sem informação & 0 & $5(1,3)$ & \\
\hline IADL, média (DP) & $3,7(2)$ & $3,4(1,9)$ & 0,190 \\
\hline PSMS, média (DP) & $3,7(2)$ & $3,7(1,8)$ & 0,935 \\
\hline \multicolumn{4}{|l|}{ Cuidadores informais } \\
\hline Idade, anos, média (DP) & $64,9(15,0)$ & $66,7(12,9)$ & 0,318 \\
\hline Sexo, feminino, n (\%) & $44(66,7)$ & $255(66,4)$ & 0,967 \\
\hline Educação, anos, média (DP) & $9(6,3)$ & $12,4(3,8)$ & 0,001 \\
\hline Coabitação com a pessoa com demência, n (\%) & $56(84,8)$ & $267(69,5)$ & 0,011 \\
\hline Relação com a pessoa com demência, n (\%) & & & 0,447 \\
\hline Cônjuge/regime marital & $40(60,6)$ & $248(64,4)$ & \\
\hline Filho, filha & $20(30,3)$ & $117(30,4)$ & \\
\hline Outro familiar (ex: nora, irmã) & $6(9,1)$ & $20(5,2)$ & \\
\hline Ansiedade (HADS), média (DP) & $6,5(3,9)$ & $6,2(3,8)$ & 0,452 \\
\hline Depressão (HADS), média (DP) & $6,4(4,4)$ & $4,5(3,4)$ & 0,001 \\
\hline RSS total, média (DP) & $22,3(11,5)$ & $21,1(10,8)$ & 0,432 \\
\hline Sofrimento emocional & $10,5(4,8)$ & $9,2(4,9)$ & 0,038 \\
\hline Sofrimento social & $7,3(5,3)$ & $7,6(4,8)$ & 0,318 \\
\hline Sentimentos negativos & $4,4(2,9)$ & $4,4(2,9)$ & 0,262 \\
\hline Tempo de perseverança, n (\%) & & & 0,544 \\
\hline Menos do que 1 semana & 0 & 0 & \\
\hline Mais do que 1 semana, menos do que 1 mês & 0 & $5(1,3)$ & \\
\hline Mais do que 1 mês, menos do que 6 meses & $4(6,3)$ & $19(5,0)$ & \\
\hline Mais do que 6 meses, menos do que 1 ano & $7(11,1)$ & $30(7,9)$ & \\
\hline Mais do que 1 ano, menos do que 2 anos & $7(9,5)$ & $59(15,6)$ & \\
\hline Mais do que 2 anos & $48(73,0)$ & $265(70,1)$ & \\
\hline SOC-13, média (DP) & $64(11,1)$ & $67,7(10,9)$ & 0,012 \\
\hline
\end{tabular}

CDR: Clinical Dementia Rating Scale; MMSE: Mini Mental State Examination; NPI: Neuropsychiatric Inventory; IADL: Instrumental Activities of Daily Living; PSMS: Physical Self-Maintenance Scale; HADS: Hospital Anxiety Depression Rating Scale; RSS: Relative Stress Scale; SOC-13: Sense of Coherence Scale-13. ${ }^{a}$ Dados não publicados. Nota: em Kerpershoek, Vugt, Wolfs, et al. $(2017)^{47}$ os dados referem-se à amostra global EU-Actifcare $(n=451)$. ${ }^{\mathrm{b}}$ Índice de Charlson - ausência de comorbilidade: 0 -1 patologias) / comorbilidade baixa: 2 patologias / comorbilidade elevada: $\geq 3$ patologias. 
Os familiares-cuidadores tinham entre 35 e 91 anos (média 64,9 ), sendo maioritariamente mulheres (cerca de $2 / 3$ ), em relação conjugal com a pessoa com demência $(60,6 \%)$ e/ ou coabitando com ela (84,8\%). Alguns não tinham escolaridade $(13,6 \%), 48,5 \%$ tinha o ensino básico, $16,7 \%$ o secundário e $21,2 \%$ um grau superior.

Aplicando a HADS aos familiares-cuidadores identificámos $6(9,1 \%)$ casos de depressão coexistindo com ansiedade, 7 (10,6\%) de depressão sem ansiedade e $3(4,5 \%)$ de ansiedade sem depressão (quadros prováveis: limiar 10/11). Estes familiares-cuidadores com ansiedade e/ou depressão (16 casos; 24,2\%) reportaram, relativamente aos restantes 50, maior sobrecarga familiar (RSS: mediana 50,2 vs 28,2; $p<0,001$ ). Não apurámos diferenças significativas nas necessidades dos doentes correspondentes. Quanto às necessidades próprias dos cuidadores, houve uma associação significativa entre necessidades de 'sofrimento psicológico' (mas não de 'informação sobre demên- cia') e ansiedade e/ou depressão prováveis $(p=0,004)$.

\section{Necessidades de cuidados \\ Total de necessidades de cuidados e diferenças de perspetiva}

A Tabela 3 apresenta, em primeiro lugar, as necessidades não-cobertas (item a item) na perspetiva do doente e do familiar-cuidador. A concordância entre estas duas perspetivas foi inexistente ou fraca na maioria dos itens. Encontrámos níveis moderados-bons apenas em 'alimentação', 'mobilidade/quedas', 'visão/audição/comunicação', 'cuidados pessoais' e 'cuidados a outras pessoas'. As necessidades não-cobertas mais frequentemente identificadas pelos próprios doentes foram relativas a 'sofrimento psicológico' (19,7\%), 'informação' (18,2\%), 'memória' (18,5\%), 'companhia' (18,5\%) e 'visão/audição/comunicação' (16,7\%). Os familiares-cuidadores destacaram, nos respetivos doentes, necessidades não-cobertas de 'atividades diárias' (27,3\%),

Tabela 3 - Necessidades de cuidados não-cobertas (perspetivas da pessoa com demência e do cuidador informal) e total de necessidades de cuidados (incluindo a perspetiva do avaliador)

\begin{tabular}{|c|c|c|c|c|}
\hline Necessidades não-cobertas, n (\%) & Pessoa com demência & Cuidador informal & Concordância kappa ${ }^{a}$ & Valor $p^{b}$ \\
\hline Alojamento & $1(1,5)$ & $1(1,5)$ & $-0,016$ & 0,865 \\
\hline Cuidados com a casa & $2(3)$ & $1(1,5)$ & 0,357 & $<0,001$ \\
\hline Alimentação & $0(0)$ & $1(1,5)$ & 0,643 & $<0,001$ \\
\hline Cuidados pessoais & $1(1,5)$ & $0(0)$ & 0,507 & $<0,001$ \\
\hline Cuidados prestados a outras pessoas & $0(0)$ & $0(0)$ & 0,476 & $<0,001$ \\
\hline Atividades diárias & $4(6,1)$ & $18(27,3)$ & 0,224 & 0,001 \\
\hline Memória & $12(18,5)$ & $8(12,1)$ & 0,041 & 0,470 \\
\hline Visão / audição / comunicação & $11(16,7)$ & $4(6,1)$ & 0,572 & $<0,001$ \\
\hline Mobilidade / quedas & $2(3)$ & $2(3)$ & 0,626 & $<0,001$ \\
\hline Continência de esfíncteres & $5(7,8)$ & $5(7,8)$ & 0,359 & $<0,001$ \\
\hline Saúde física & $1(1,5)$ & $4(6,2)$ & 0,061 & 0,333 \\
\hline Medicamento ou tóxicos & $1(1,5)$ & $3(4,6)$ & 0,203 & 0,012 \\
\hline Sintomas psicóticos & $1(1,5)$ & $2(3)$ & 0,134 & 0,052 \\
\hline Sofrimento psicológico & $13(19,7)$ & $10(15,2)$ & 0,312 & $<0,001$ \\
\hline Informação (sobre a doença e tratamento) & $12(18,2)$ & $4(7)$ & 0,039 & 0,721 \\
\hline Segurança pessoal (conduta parasuicidária) & $2(3)$ & $1(1,5)$ & 0,313 & 0,001 \\
\hline Segurança pessoal (risco não intencional) & $2(3)$ & $1(1,5)$ & 0,299 & 0,002 \\
\hline Abuso / negligência & $0(0)$ & $0(0)$ & -- & -- \\
\hline Comportamento & $0(0)$ & $2(3)$ & -- & -- \\
\hline Álcool & $0(0)$ & $0(0)$ & -- & -- \\
\hline Companhia & $12(18,5)$ & $10(15,6)$ & 0,288 & 0,003 \\
\hline Relações íntimas & $5(7,7)$ & $3(4,6)$ & $-0,064$ & 0,564 \\
\hline Dinheiro / economias & $0(0)$ & $1(1,5)$ & 0,261 & 0,003 \\
\hline Benefícios sociais & $1(1,5)$ & $8(12,1)$ & 0,029 & 0,733 \\
\hline Total de necessidades, média (DP) & Pessoa com demência & Cuidador informal & Avaliador & Valor $p^{c}$ \\
\hline Necessidades não-cobertas & $1,3(1,8)$ & $1,4(1,4)$ & $1,1(1,7)$ & 0,238 \\
\hline Necessidades cobertas & $5,0(2,6)$ & $8,0(2,7)$ & $7,7(2,8)$ & $<0,001$ \\
\hline Necessidades globais (cobertas ou não) & $6,3(2,7)$ & $9,4(3,0)$ & $8,9(3,1)$ & $<0,001$ \\
\hline
\end{tabular}

a $O$ coeficiente kappa para cada item foi calculado para determinar a concordância entre a pessoa com demência e o respetivo cuidador quanto às necessidades não-cobertas da pessoa com demência. ${ }^{b} \mathrm{O}$ valor $p$ refere-se às diferenças entre as necessidades não-cobertas (item a item) nas perspetivas do doente e do familiar-cuidador. ${ }^{\circ} \mathrm{O}$ valor $p$ refere-se às diferenças entre o total de necessidades não-cobertas, cobertas e necessidades globais nas perspetivas do doente, familiar-cuidador e avaliador (teste de Kruskal-Wallis). 
'sofrimento psicológico' (15,2\%), 'companhia' (15,6\%), 'memória' $(12,1 \%)$ e 'benefícios sociais' $(12,1 \%)$.

Comparámos, ainda na Tabela 3, o total de necessidades nas perspetivas dos doentes, familiares-cuidadores e avaliadores. Não encontrámos diferenças significativas nas necessidades não-cobertas. As necessidades cobertas (e totais) foram referidas em número menor pelos doentes, em comparação com familiares-cuidadores e avaliadores $(p<0,001)$. Segundo os avaliadores, o número de necessidades não-cobertas por pessoa variou entre 0 e 8 , sendo maior que 2 em 9 (13,6\%) participantes.

\section{Necessidades de cuidados na perspetiva integrado- ra dos avaliadores \\ Apresentamos seguidamente as necessidades de cui- dados das pessoas com demência e dos familiares-cui- dadores (item a item), segundo os avaliadores (Tabela 4). As necessidades não-cobertas mais comuns nos doentes}

(média 1,1; DP = 1,7) foram: 'companhia' $(22,7 \%)$, 'sofrimento psicológico' (19,7\%) e 'atividades diárias' (13,6\%). Embora 33 (50,0\%) não tivessem necessidades não-cobertas, $9(14,0 \%)$ apresentavam pelo menos três. Quase metade dos familiares-cuidadores apresentava necessidades não-cobertas em 'sofrimento psicológico' (43,9\%), embora quanto a 'informação' os avaliadores apenas as referissem em $12,1 \%$ dos casos (Tabela 4). Na sua perspetiva própria, $25,8 \%$ dos familiares-cuidadores havia reportado necessidades não-cobertas em 'sofrimento psicológico' e 19,7\% necessidades de 'informação'.

\section{Utilização dos cuidados formais e informais}

No mês anterior às entrevistas, 44 destas pessoas com demência $(66,7 \%)$ não utilizaram serviços formais (e.g. enfermagem/cuidados domiciliários, entrega de refeições, centro de dia). Os restantes $33,3 \%$ receberam cuidados formais compatíveis com os critérios de inclusão (e.g.

Tabela 4 - Necessidades de cuidados das pessoas com demência e dos cuidadores informais, segundo a perspetiva integradora dos avaliadores

\begin{tabular}{|c|c|c|c|c|}
\hline \multirow[b]{2}{*}{ Itens/domínios } & \multicolumn{4}{|c|}{ Necessidades identificadas } \\
\hline & $\begin{array}{c}\text { Sem } \\
\text { necessidade }\end{array}$ & $\begin{array}{c}\text { Necessidade } \\
\text { coberta }\end{array}$ & $\begin{array}{c}\text { Necessidade } \\
\text { não-coberta }\end{array}$ & $\begin{array}{c}\text { Sem } \\
\text { informação }\end{array}$ \\
\hline \multicolumn{5}{|l|}{ Necessidades das pessoas com demência, n (\%) } \\
\hline Alojamento & $65(98,5)$ & $1(1,5)$ & - & - \\
\hline Cuidados com a casa & $10(15,2)$ & $55(83,3)$ & $1(1,5)$ & - \\
\hline Alimentação & $11(16,7)$ & $53(80,3)$ & $1(1,5)$ & $1(1,5)$ \\
\hline Cuidados pessoais & $26(39,4)$ & $39(59,1)$ & - & $1(1,5)$ \\
\hline Cuidados prestados a outras pessoas & $59(89,4)$ & $6(9,1)$ & $1(1,5)$ & - \\
\hline Atividades diárias & $37(56,1)$ & $19(28,8)$ & $9(13,6)$ & $1(1,5)$ \\
\hline Memória & - & $61(92,4)$ & $3(4,5)$ & $2(3,0)$ \\
\hline Visão / audição / comunicação & $47(71,2)$ & $11(16,7)$ & $4(6,1)$ & $4(6,1)$ \\
\hline Mobilidade / quedas & $45(68,2)$ & $18(27,3)$ & $2(3,0)$ & $1(1,5)$ \\
\hline Continência de esfíncteres & $47(71,2)$ & $9(13,6)$ & $5(7,6)$ & $5(7,6)$ \\
\hline Saúde física & $2(3,0)$ & $62(93,9)$ & $1(1,5)$ & $1(1,5)$ \\
\hline Medicamento ou tóxicos & $7(10,6)$ & $52(78,8)$ & $2(3,0)$ & $5(7,6)$ \\
\hline Sintomas psicóticos & $57(86,4)$ & $5(7,6)$ & $1(1,5)$ & $3(4,5)$ \\
\hline Sofrimento psicológico & $37(56,1)$ & $12(18,2)$ & $13(19,7)$ & $4(6,1)$ \\
\hline Informação (sobre a doença e tratamento) & $57(86,4)$ & - & $2(3,0)$ & $7(10,6)$ \\
\hline Segurança pessoal (conduta parasuicidária) & $61(92,4)$ & $1(1,5)$ & $2(3,0)$ & $2(3,0)$ \\
\hline Segurança pessoal (risco não intencional) & $25(37,9)$ & $31(47,0)$ & $4(6,1)$ & $6(9,1)$ \\
\hline Abuso / negligência & $63(95,5)$ & - & $1(1,5)$ & $2(3,0)$ \\
\hline Comportamento & $55(80,3)$ & $8(12,1)$ & $1(1,5)$ & $2(3,0)$ \\
\hline Álcool & $64(97,0)$ & - & $2(3,0)$ & - \\
\hline Companhia & $43(65,2)$ & $5(7,6)$ & $15(22,7)$ & $3(4,5)$ \\
\hline Relações íntimas & $61(90,9)$ & $1(1,5)$ & $2(3,0)$ & $2(3,0)$ \\
\hline Dinheiro / economias & $6(13,6)$ & $58(87,9)$ & $1(1,5)$ & $1(1,5)$ \\
\hline Benefícios sociais & $44(66,7)$ & $4(6,1)$ & $2(3,0)$ & $16(24,2)$ \\
\hline \multicolumn{5}{|l|}{ Necessidades dos cuidadores informais, $n$ (\%) } \\
\hline Necessidades de informação & $51(77,3)$ & $3(4,5)$ & $8(12,1)$ & $4(6,1)$ \\
\hline Sofrimento psicológico & $24(36,4)$ & $11(16,7)$ & $29(43,9)$ & $2(3,0)$ \\
\hline
\end{tabular}


enfermagem/cuidados domiciliários, mas por outras doenças; empregadas domésticas antigas não prestando apoio significativo na incapacidade associada à demência) (Tabela 5).

Dos 44 casos 'não utilizadores', 20 (i.e. 30,3\% do total) considerariam a hipótese de recorrer a algum tipo de cuidados formais tal como definidos no protocolo. A razão mais frequentemente indicada para a sua não utilização foi a recusa $(33,4 \%)$ da pessoa com demência, do cuidador ou de ambos, sobretudo no respeitante a centros de dia, entrega de refeições ou admissão temporária/de alívio num hospital ou lar. Os participantes manifestaram relutância na utilização destes serviços alegando falta de qualidade e associando-os a "dependência" e "declínio" (sic). Também o desconhecimento $(25,8 \%)$ levou alguns doentes e/ou familiares-cuidadores a não considerarem serviços aos quais, conforme admitido nas entrevistas, poderiam atribuir algum interesse: e.g. admissão temporária/de alívio, grupos de apoio para cuidadores, estimulação cognitiva. As restantes razões $(40,8 \%)$ para a não utilização distribuíram-se por categorias distintas (e.g. tempos de espera; inexistência; distância; inacessibilidade financeira).

Nos casos evidenciando as necessidades não-cobertas mais comuns ('companhia', 'sofrimento psicológico' e 'atividades diárias'), o apoio recebido dos serviços locais para lidar com estes problemas (CANE/secção III) era frequentemente menor que o julgado necessário pelo avaliador. Por exemplo, nenhum doente com necessidades não-cobertas de 'atividades diárias' recebia apoio, mas 88,8\% necessitaria assistência pequena-moderada. Note-se que $49(74,2 \%)$ registavam consulta(s) médicas no mês anterior (não consideradas 'cuidados formais' neste projeto).

Por último, quanto à prestação de cuidados informais, a moda foi 60 minutos e a mediana 150 minutos/dia (sobretudo para assistência nas atividades instrumentais de vida diária: 93 minutos/dia). Embora em 54 dos casos (81,8\%) houvesse pelo menos mais um cuidador informal envolvido, a maioria dos cuidadores principais $(57,6 \%)$ referiu ter assegurado mais de $80 \%$ dos cuidados.

\section{DISCUSSÃO}

O projeto EU-Actifcare centrou-se no acesso e utilização de serviços como centros de dia ou apoio domiciliário. Considerando pessoas com demência e familiares-cuidadores numa visão tendencialmente sistémica, incluiu o estudo observacional de uma amostra europeia de dimensão apreciável, criteriosamente selecionada. Reportamos uma avaliação detalhada de necessidades e recurso a serviços, na subamostra de pessoas com demência ligeira a moderada vivendo na comunidade, em Portugal.

Tabela 5 - Utilização dos cuidados formais e informais

\begin{tabular}{|c|c|}
\hline \multicolumn{2}{|l|}{ Utilização dos cuidados formais ${ }^{a}$} \\
\hline Sem utilização dos cuidados formais, n (\%) & $44(66,7)$ \\
\hline Com utilização dos cuidados formais, $n$ (\%) & $22(33,3)$ \\
\hline Enfermeiro ao domicílio & $3(4,5)$ \\
\hline Ajuda doméstica & $16(24,2)$ \\
\hline Serviço de entrega de refeições & $5(7,6)$ \\
\hline Cuidados de dia & - \\
\hline Transporte (relacionado com cuidados de saúde) & - \\
\hline Outros & $1(1,5)$ \\
\hline \multicolumn{2}{|l|}{ Utilização dos cuidados informais ${ }^{a}$} \\
\hline \multicolumn{2}{|c|}{ Tempo despendido pelos cuidadores, minutos/dia, mediana (min - máx) } \\
\hline Atividades de vida diária & $13(0-240)$ \\
\hline Atividades instrumentais de vida diária & $93(6-300)$ \\
\hline Supervisão & $16(0-480)$ \\
\hline Total & $150(6-870)$ \\
\hline \multicolumn{2}{|l|}{ Outros cuidadores informais envolvidos, n (\%) } \\
\hline 0 & $12(18,2)$ \\
\hline 1 & $25(37,9)$ \\
\hline 2 & $14(21,2)$ \\
\hline 3 ou mais & $15(22,7)$ \\
\hline \multicolumn{2}{|l|}{ Nível de contribuição na prestação de cuidados ${ }^{b}, \mathrm{n}(\%)$} \\
\hline $21 \%-40 \%$ & $1(1,5)$ \\
\hline $41 \%-60 \%$ & $5(7,6)$ \\
\hline $61 \%-80 \%$ & $22(33,3)$ \\
\hline $81 \%-100 \%$ & $38(57,6)$ \\
\hline
\end{tabular}

a Nos últimos 30 dias; ${ }^{b}$ Entre todos os cuidadores informais envolvidos, avalia-se qual o nível de contribuição do cuidador principal entrevistado 


\section{Resultados principais e comparação com outros estu- os}

Na perspetiva dos avaliadores, os participantes apresentavam, em média, uma necessidade não-coberta. Estas necessidades eram, principalmente, de 'companhia' (22,7\%), 'sofrimento psicológico' $(19,7 \%)$ e 'atividades diárias' $(13,6 \%)$ (sugerindo insuficiências dos cuidados informais e/ou indicação parcial para cuidados formais, pois os serviços poderiam equacionar respostas nestes domínios). Nos participantes não-portugueses da amostra EU-Actifca$\mathrm{re}^{47}$, as necessidades não-cobertas mais frequentes eram em 'atividades diárias' (32,1\%), 'companhia' (28,7\%) e 'memória' $(15,1 \%)$. As necessidades cobertas mais frequentes foram, em Portugal, 'saúde física' (93,9\%), 'memória' $(92,4 \%)$ e 'dinheiro/economias' $(87,9 \%)$. Nos participantes não-portugueses, estas necessidades foram inferiores em 'saúde física' (65,4\%) e 'memória' (82,6\%), e semelhantes para 'dinheiro/economias' (83,2\%). Porém, a comparação dos níveis de necessidades nos países que integraram o EU-Actifcare é limitada pelo facto de lidarmos com amostras de conveniência. O mesmo se aplica à comparação de outros resultados do nosso estudo entre os países participantes, ou à comparação dos níveis de necessidades com resultados de outros estudos.

Ainda assim, genericamente, os nossos achados assemelham-se aos de trabalhos internacionais, e.g. Miranda-Castillo et al, em que as necessidades não-cobertas mais frequentes eram em 'atividades diárias' (50,7\%), 'companhia' (39,5\%) e 'sofrimento psicológico' (30,9\%). ${ }^{48}$ Mas realçamos que só em $5 \%$ dos nossos casos havia necessidades não-cobertas de 'memória' (estavam quase sempre cobertas), sendo esta percentagem habitualmente maior em pessoas com demência em hospitais de dia, ${ }^{4}$ internamento hospitalar ${ }^{49}$ ou lares ${ }^{4,50,51}$ (ou mesmo em populações geriátricas heterogéneas no diagnóstico neuropsiquiátrico). ${ }^{52}$ Noutros estudos nacionais, as necessidades não-cobertas foram sobretudo de 'sofrimento psicológico', 'atividades diárias', 'companhia' e 'memória'9 ou 'sofrimento psicológico', 'atividades diárias' e 'benefícios'. ${ }^{10} \mathrm{O}$ interesse dos nossos resultados releva da especificidade da amostra (demência ligeira a moderada, sem cuidados formais na comunidade), invulgar na literatura.

Há evidência de divergências relativas nos resultados da avaliação das necessidades de pessoas com demência (comparando e.g., a perspetiva dos próprios e dos seus familiares-cuidadores). ${ }^{7,51,53}$ Nesta pequena amostra portuguesa, esta divergência foi mais evidente em 'alojamento', 'memória', 'saúde física', 'informação', 'relações íntimas' e 'benefícios sociais'. Porém, não registámos diferença significativa entre doentes e familiares-cuidadores no total das necessidades não-cobertas, enquanto na amostra global EU-Actifcare o nível médio destas necessidades foi quase o dobro na perspetiva do familiar-cuidador. ${ }^{47}$ Este resultado poderá refletir um mero efeito de amostragem ou alguma influência da menor escolaridade dos nossos familiares-cuidadores. Já quanto às necessidades cobertas, registámos níveis significativamente superiores na perspetiva dos familiares, tal como aconteceu na amostra global. ${ }^{47}$ Em todo o caso, nenhuma destas comparações 'internacionais' deve ser hipervalorizada (cf. Limitações). Independentemente da difícil interpretação de algumas tendências globais (nível amostral), as diferenças de perspetiva entre doentes, familiares-cuidadores e avaliadores são importantes, em termos clínicos (nível individual) e de avaliação dos serviços, justificando avaliações sistemáticas das necessidades de cuidados. ${ }^{4}$

Quanto às necessidades próprias dos familiares-cuidadores, as necessidades de 'sofrimento psicológico' foram superiores ao habitual na literatura nacional ${ }^{9,10,14}$ e internacional $^{5}$ (quase metade apresentava este tipo de necessidades não-cobertas). Por outro lado, apenas um quarto tinha morbilidade psiquiátrica minor 'provável' (HADS). Embora a diferença nas proporções de necessidades e psicopatologia fosse menor considerando casos HADS 'possíveis', confirma-se que identificar 'necessidades' difere conceptualmente de estabelecer diagnósticos clínicos. Também os restantes resultados destes familiares-cuidadores são consistentes com a avaliação de necessidades. Face aos dados da literatura, ${ }^{35,54}$ apresentavam sobrecarga objetiva (mediana de quase 150 minutos/dia em prestação de cuidados) e subjetiva (RSS total: média 22,3; DP = 11,5). Quase um terço sentia-se incapaz de continuar a prestar cuidados, nas mesmas condições, por mais de dois anos. Outros estudos relataram perseverança menor, mas em amostras incluindo doentes mais graves. ${ }^{55}$

Em suma, o nível e o tipo de necessidades sugerem, numa proporção de participantes, acesso não atempado aos cuidados formais na comunidade. A maioria não havia utilizado serviços formais no mês anterior e, quando tal se verificou, as razões não estavam diretamente ligadas à demência. Nesta avaliação inicial da coorte, parte dos problemas de acesso/utilização dos serviços radicava em atitudes negativas de utentes e/ou familiares (e.g. recusa, estigma) e não tanto em barreiras estruturais (e.g. oferta e proximidade dos cuidados) ou organizacionais (e.g. tempos de espera ou referenciação). Assim, mesmo numa amostra intencionalmente selecionada para não precisar de cuidados formais relevantes (CDR $<2$ ), e com apoio clínico comprovado em muitos casos, verificámos necessidades não-cobertas e problemas de procura, acessibilidade ou utilização dos cuidados formais na comunidade.

\section{Especificidades da amostra e dificuldades no recruta- mento}

Selecionámos uma amostra relativamente comum, em termos clínico-funcionais, de pessoas com demência ligeira a moderada, na comunidade. Apesar de a proporção de participantes com nível de ensino secundário ou superior exceder a habitual nestas idades ${ }^{56}$, recrutámos diversificadamente, em termos geográficos, sociais e de serviços. A maioria dos participantes vivia em Lisboa e Setúbal, mas $27,3 \%$ eram do interior do país, incluindo zonas urbanas e rurais. As pontuações no MMSE foram significativamente mais baixas que as dos restantes participantes no 
EU-Actifcare. Para tal poderá ter contribuído a menor escolaridade dos participantes portugueses, com a agravante de termos usado a tradução oficial, não adaptada para Portugal. Quanto aos familiares-cuidadores, a predominância de mulheres e cônjuges é comum nestas populações, designadamente em Portugal (onde o número de participantes vivendo com o doente foi, como esperado, significativamente superior ao dos restantes países). Também os resultados da sua avaliação psicossocial e psicopatológica corroboram a validade interna e, parcialmente, externa do estudo (cf. secção anterior).

A dimensão reduzida da amostra $(n=66)$ não se deveu aos problemas de recrutamento: no EU-Actifcare o objetivo era de 50 díades por país e Portugal ultrapassou esse número. Porém, foi necessário esforço da equipa face às dificuldades iniciais (aliás esperadas, dada a realidade nacional de atraso/inexistência no diagnóstico de demência e/ou utilização dos serviços formais). Com efeito, os critérios eram intencionalmente restritivos, para incluir doentes já com diagnóstico de demência mas ainda sem níveis de incapacidade que implicassem taxativamente cuidados formais. Em Portugal, muitos doentes têm diagnóstico tardio, nem sempre reconhecido em serviços onde sejam seguidos por outros motivos (e.g. cuidados primários, serviços de medicina/cirurgia). ${ }^{2,22,23}$ Também muitos doentes com diagnóstico confirmado foram excluídos por já terem ajuda formal considerável para problemas decorrentes da demência. Persistindo, com assimetrias regionais, a escassez de respostas comunitárias (centros de dia para a demência ou, talvez em menor grau, apoio domiciliário adequado), muitos contavam com empregadas domésticas como cuidadoras formais não qualificadas ou, poderiam estar institucionalizados precocemente.

\section{Limitações do estudo}

De acordo com o protocolo EU-Actifcare ${ }^{31}$ tal como nos restantes países, os resultados não são necessariamente generalizáveis à população de pessoas com demência em Portugal, nem mesmo ao subgrupo de doentes com demência ligeira a moderada. Contudo, para reduzir o viés de seleção, diversificámos a proveniência dos participantes quanto a regiões, serviços de saúde e sociais. Trata-se de uma amostra relativamente típica, com eventual desvio no sentido de maior escolaridade.

A menor literacia de alguns participantes poderia ter comprometido as respetivas avaliações. Contudo, os entrevistadores foram treinados especificamente e algumas entrevistas divididas em momentos próximos, intercalando interações informais para diminuir a sobrecarga avaliativa e preservar a qualidade dos dados. Reconhece-se um possível viés pelo uso da versão oficial do MMSE, como discutido acima; porém, as pontuações não foram usadas na análise.

A reduzida dimensão da amostra limita, de resto, uma análise multivariável detalhada. Esta tem sido feita com a amostra global (451 díades) ${ }^{47}$ e será parcialmente explorada no estudo longitudinal português. Estudos futuros deverão abordar, por exemplo, o papel das discrepâncias regionais ou da prestação de cuidados informais, ou particularidades nas demências de início precoce.

\section{Implicações do estudo em termos de políticas e servi- ços}

A documentação das dificuldades no acesso e utilização dos serviços formais poderá pressionar a implementação de políticas específicas. O esforço do projeto EU-Actifcare para sistematizar estas dificuldades envolveu revisões da literatura, ${ }^{57}$ explorações qualitativas com pessoas com demência, familiares-cuidadores e profissionais, ${ }^{58}$ bem como com administradores/decisores políticos. ${ }^{59}$ Numa segunda fase, esperamos que os resultados do estudo longitudinal permitam compreender melhor os determinantes do recurso aos serviços, potenciando facilitadores e diminuindo barreiras. No rescaldo do projeto, surgiram recomendações de boas práticas para melhorar o acesso/utilização dos cuidados formais. Deverá discutir-se a sua aplicabilidade nacional, nomeadamente quanto ao papel dos cuidados de saúde primários. ${ }^{23,60}$

O acesso atempado e a utilização adequada dos serviços na comunidade poderão diminuir as necessidades não-cobertas de pessoas com demência e dos seus cuidadores informais, bem como a sobrecarga das famílias.

\section{CONCLUSÃO}

Estes primeiros resultados relativos à coorte portuguesa Actifcare sugerem dificuldades relevantes no recurso a cuidados formais na comunidade em pessoas com demência ligeira a moderada e seus familiares-cuidadores. A avaliação revelou necessidades não-cobertas relevantes em áreas específicas (principalmente 'companhia', 'sofrimento psicológico' e 'atividades diárias'), a par de repercussões familiares importantes. Havendo problemas no acesso/utilização de alguns cuidados formais na comunidade, a implementação de boas práticas específicas poderá colmatar muitas necessidades destas populações.

\section{AGRADECIMENTOS}

O consórcio Actifcare é coordenado na Maastricht University (Frans Verhey, Marjolein de Vugt, coordenadores científicos) e composto, além dos autores deste artigo, por: Claire Wolfs, Ron Handels, Liselot Kerpershoek (Maastricht University, Holanda); Gabriele Meyer, Astrid Stephan, Anja Bieber, Anja Broda, Gabriele Bartoszek (Martin Luther University Halle-Wittenberg, Alemanha); Bob Woods, Hannah Jelley (Bangor University, Reino Unido); Martin Orrell (Nottingham Institute of Mental Health, Reino Unido); Anders Wimo, Anders Sköldunger, Britt-Marie Sjölund (Karolinska Institutet, Suécia); Knut Engedal, Geir Selbaek, Mona Michelet, Janne Rosvik, Siren Eriksen (Vestfold Hospital Trust/ Oslo University Noruega); Kate Irving, Louise Hopper, Rachael Joyce (Dublin City University, Irlanda); Orazio Zanetti, Elisa Portolani (IRCCS Centro S. Giovanni di Dio - Fatebenefratelli, Itália).

Colaboraram também no trabalho de campo, como 
entrevistadores: Ana Nunes, Ana Teixeira, Filipa Barreiros, Helena Amaro, Liliete Barrocas, Mariana Cunha, Patrícia Ramalho.

Agradecemos às Direções dos serviços de saúde envolvidos (ACES Alentejo Central, Centro de Saúde de Mora, USF Fernão Ferro mais, USF Marginal, Serviço de Neurologia e Serviço de Psiquiatria do Hospital Egas Moniz/CHLO, Hospital do Mar - Luz Saúde), à Alzheimer Portugal, à Associação de Beneficência Popular de Gouveia e à Santa Casa da Misericórdia de Mora. Agradecemos ainda a Isabel Santana (CHUC/Universidade de Coimbra), Albino Oliveira-Maia e Teresa Ventura (NMS/FCM, UNL) a referenciação de casos, nas zonas de Gouveia e Lisboa.

\section{PROTECÇÃO DE PESSOAS E ANIMAIS}

Os autores declaram que os procedimentos seguidos estavam de acordo com os regulamentos estabelecidos pelos responsáveis das Comissões de Investigação Clínica e Ética NMS/Faculdade de Ciências Médicas; Centro Hospitalar de Lisboa Ocidental; ARS Lisboa e Vale do Tejo; ARS Alentejo, e pela Comissão Nacional de Protecção de

\section{REFERÊNCIAS}

1. Prince M, Wimo A, Guerchet M, Ali G, Wu YT, Prina M. World Alzheimer Report 2015: The Global Impact of Dementia. London: Alzheimer's Disease International; 2015.

2. Santana I, Farinha F, Freitas S, Rodrigues V, Carvalho Á. Epidemiologia da demência e da doença de Alzheimer em Portugal: Estimativas da prevalência e dos encargos financeiros com a medicação. Acta Med Port. 2015;28:182-8.

3. Gonçalves-Pereira M, Cardoso A, Verdelho A, Alves da Silva J, Caldas de Almeida M, Fernandes A, et al. The prevalence of dementia in a Portuguese community sample: a 10/66 Dementia Research Group study. BMC Geriatr. 2017;17:261.

4. Orrell M, Hancock G. CANE: Camberwell Assessment of Need for the Elderly. London: Gaskell; 2004.

5. Reynolds T, Thornicroft G, Abas M, Woods B. Camberwell Assessment of Need for the Elderly: Development, validity and reliability. $\mathrm{Br} \mathrm{J}$ Psychiatry. 2000;176:444-52.

6. Wright J, Williams R, Wilkinson JR. Development and importance of health needs assessment. Br Med J. 1998;316:1310-3.

7. Miranda-Castillo C, Woods B, Orrell M. The needs of people with dementia living at home from user, caregiver and professional perspectives: a cross-sectional survey. BMC Health Serv Res. 2013;13:1-10.

8. Gonçalves-Pereira M, Fernandes L, Leuschner A, Barreto J, Falcão D, Firmino H. Versão portuguesa do CANE (Camberwell Assessment of Need for the Elderly): desenvolvimento e dados preliminares. Rev Port Saúde Pública. 2007;25:7-18.

9. Fernandes L, Gonçalves-Pereira M, Leuschner A, Martins S, Sobral M, Azevedo LF, et al. Validation study of the Camberwell Assessment of Need for the Elderly (CANE) in Portugal. Int Psychogeriatr. 2009;21:94102.

10. Passos J, Fonte A, Dias CC, Fernandes L. The needs of older people with mental health problems in a Portuguese psychiatric setting. Int Psychogeriatrics. 2017:1-9.

11. McCabe M, You E, Tatangelo G. Hearing their voice: a systematic review of dementia family caregivers' needs. Gerontologist. 2016;56:70-88.

12. Cheng ST. Dementia caregiver burden: a research update and critical analysis. Curr Psychiatry Rep. 2017;19:64.

13. Borsje P, Hems MA, Lucassen PL, Bor H, Koopmans RT, Pot AM. Psychological distress in informal caregivers of patients with dementia in primary care: course and determinants. Fam Pract. 2016;0:1-8.

14. Gonçalves-Pereira M, Carmo I, Silva JA, Papoila AL, Mateos R, Zarit SH. Caregiving experiences and knowledge about dementia in Portuguese clinical outpatient settings. Int Psychogeriatrics. 2010;22:270-80.

15. Sequeira C. Difficulties, coping strategies, satisfaction and burden in informal Portuguese caregivers. J Clin Nurs. 2013;22:491-500.

16. Prince M, Comas-Herrera A, Knapp M, Guerchet M, Karagiannidou M.
Dados, bem como de acordo com a Declaração de Helsínquia da Associação Médica Mundial.

\section{CONFIDENCIALIDADE DOS DADOS}

Os autores declaram ter seguido os protocolos do seu centro de trabalho acerca da publicação de dados.

\section{CONFLITOS DE INTERESSE}

Os autores declaram não ter qualquer conflito de interesse relativamente ao presente artigo.

\section{FONTES DE FINANCIAMENTO}

O projeto Actifcare em Portugal foi financiado pela Fundação para a Ciência e a Tecnologia (FCT-JPND-HC-0001/2012), com o apoio da iniciativa de Programação Conjunta da União Europeia - Investigação em Doenças Neurodegenerativas, JPND (EU Joint Programme - Neurodegenerative Disease Research), JPND/2013/2.

Maria J. Marques foi apoiada pela FCT (PD/ BD/128011/2016) e pelo Fundo Social Europeu através do Programa Operacional Potencial Humano.

World Alzheimer Report 2016: Improving Healthcare for People Living with Dementia. London: Alzheimer's Disease International; 2016.

17. Fernandes $L$, Mateos $R$, Engedal $K$, von Gunten $A$, Stek $M L$, Ramakrishnan $A$, et al. The state of psychogeriatrics in Europe: Challenges and opportunities in six European countries. Int Psychogeriatrics. 2015;27:1243-6.

18. De Vugt ME, Verhey FR. The impact of early dementia diagnosis and intervention on informal caregivers. Prog Neurobiol. 2013;110:54-62.

19. Dubois B, Padovani A, Scheltens P, Rossi A, Dell'Agnello G. Timely diagnosis for Alzheimer's Disease: A literature review on benefits and challenges. J Alzheimer's Dis JAD. 2016;49:617-31.

20. Brodaty $\mathrm{H}$, Thomson $\mathrm{C}$, Thompson $\mathrm{C}$, Fine $\mathrm{M}$. Why caregivers of people with dementia and memory loss don't use services. Int J Geriatr Psychiatry. 2005;20:537-46.

21. Werner P, Mittelman MS, Goldstein D, Heinik J. Family stigma and caregiver burden in Alzheimer's disease. Gerontologist. 2011;52:89-97.

22. Grupo de Trabalho na Área das Demências nomeado pelo Ministério da Saúde. Base para a definição de políticas públicas na área das demências. 2017. [consultado 2018 jan 12]. https://www.sns.gov. pt/2017/08/10/bases-para-a-definicao-de-politicas-publicas-na-areadas-demencias.

23. Gonçalves-Pereira M, Leuschner A. Portugal. In: Burns A, Robert P, editors. Dementia care: international perspectives. Oxford: Oxford University Press; 2019.

24. Gaugler JE, Kane RL, Kane RA, Newcomer R. Early community-based service utilization and its effects on institutionalization in dementia caregiving. Gerontologist. 2005;45:177-85.

25. Wimo A, Rönnbäck E, Larsson B, Eriksson T, Eriksson IB, Thorslund M "Misplacement" of elderly people in the caring organisation: reasons and alternatives. Arch Gerontol Geriatr. 1999;28:227-37.

26. Wolfs CA, De Vugt ME, Verkaaik M, Verkade PJ, Verhey FR. Empowered or overpowered? Service use, needs, wants and demands in elderly patients with cognitive impairments. Int J Geriatr Psychiatry. 2010;25:1006-12.

27. Andersen RM. Families' use of health services: a behavioral model of predisposing, enabling and need domponents. West Lafayette: Purdue University; 1968.

28. Andersen R. Revisiting the behavioral model and access to medical care: does it matter? J Health Soc Behav. 1995;36:1-10.

29. Andersen RM, Davidson PL. Improving access to care in America: individual and contextual indicators. In: Andersen RM, Rice TH, Kominski EF, eds. Changing the US Health Care System: key Issues in health services, policy, and management. San Francisco: Jossey-Bass; 2001. p. 3-33

30. Donath C, Ulbrecht G, Grau H, Graessel E, Schwarzkopf L, Menn P, et 
al. Health services utilization by community-dwelling dementia patients and their family caregivers. In: Health care utilization in Germany. New York: Springer New York; 2014. p. 193-219.

31. Kerpershoek L, de Vugt M, Wolfs C, Jelley H, Orrell M, Woods B, et al. Access to timely formal dementia care in Europe: Protocol of the Actifcare (ACcess to Timely Formal Care) study. BMC Health Serv Res. 2016;16:1-7.

32. American Psychiatric Association. DSM-IV-TR: Manual de Diagnóstico E Estatística Das Perturbações Mentais. $4^{\mathrm{a}}$ ed. Lisboa: Editores C; 2002.

33. Morris JC. The clinical dementia rating (CDR): current version and scoring rules. Neurology. 1993;43:2412-4.

34. Folstein MF, Folstein SE, McHugh PR. "Mini-mental state": a practical method for grading the cognitive state of patients for the clinician. J Psychiatr Res. 1975;12:189-98.

35. Wimo A, Gustavsson A, Jonsson L, Winblad B, Hsu MA, Gannon B. Application of resource utilization in dementia (RUD) instrument in a global setting. Alzheimer's Dement. 2013;9:429-35.

36. Bakker C, de Vugt ME, van Vliet D, Verhey FR, Pijnenburg YA, VernooijDassen MJ, et al. The relationship between unmet care needs in youngonset dementia and the course of neuropsychiatric symptoms: a twoyear follow-up study. Int Psychogeriatrics. 2014;26:1991-2000.

37. Kaufer DI, Cummings JL, Ketchel P, Smith V, MacMillan A, Shelley T, et al. Validation of the NPI-Q, a brief clinical form of the neuropsychiatric inventory. J Neuropsychiatry Clin Neurosci. 2000;12:233-9.

38. Charlson M, Szatrowski TP, Peterson J, Gold J. Validation of a combined psychopathology in dementia. J Clin Epidemiol. 1994;47:1245-51.

39. Lawton M, Brody EM. Assessment of older people: self-maintaining and instrumental activities of daily living. Nurs Res. 1969;19:278.

40. Zigmond AS, Snaith RP. The Hospital Anxiety and Depression Scale. Acta Psychiatr Scand. 1983;67:361-70.

41. Greene JG, Smith R, Gardiner M, Timbury GC. Measuring behavioural disturbance of elderly demented patients in the community and its effects on relatives: a factor analytic study. Age Ageing. 1982;11:121-6.

42. Kraijo H, Brouwer W, de Leeuw R, Schrijvers G, van Exel J. The perseverance time of informal carers of dementia patients: validation of a new measure to initiate transition of care at home to nursing home care. J Alzheimer's Dis. 2014;40:631-42.

43. Antonovsky A. Unraveling the mystery of health: how people manage stress and stay well. San Francisco: Jossey-Bass; 1987.

44. Bjelland I, Dahl AA, Tangen T, Neckelmann D. The validity of the hospital anxiety and depression scale an updated literature review. 2002;52:6977.

45. Pais-Ribeiro J, Silva I, Ferreira T, Martins A, Meneses R, Baltar M. Validation study of a Portuguese version of the hospital anxiety and depression scale. Psychol Health Med. 2007;12:225-37.

46. Altman DG. Practical statistics for medical research. London: Chapman and Hall; 1991.

47. Kerpershoek L, Vugt M De, Wolfs C, Woods B, Jelley H, Orrell M, et al.
Needs and quality of life of people with middle- stage dementia and their family carers from the European Actifcare study. When informal care alone may not suffice. Aging Ment Health. 2018;22:897-902.

48. Miranda-Castillo C, Woods B, Galboda K, Oomman S, Olojugba C, Orrell M. Unmet needs, quality of life and support networks of people with dementia living at home. Health Qual Life Outcomes. 2010;8:132.

49. Greaves S, Bhat M, Regan C, Qazi A, Miranda-Castillo C, Orrell M. The unmet needs of referrals to old age psychiatry liaison services. Psychogeriatria Pol. 2006;3:175-82.

50. Hancock GA, Woods B, Challis D, Orrell M. The needs of older people with dementia in residential care. Int J Geriatr Psychiatry. 2006;21:43-9.

51. Orrell M, Hancock GA, Liyanage KC, Woods B, Challis D, Hoe J. The needs of people with dementia in care homes: the perspectives of users, staff and family caregivers. Int Psychogeriatrics. 2008;20:941-51.

52. Ashaye OA, Livingston G, Orrell MW. Does standardized needs assessment improve the outcome of psychiatric day hospital care for older people? A randomized controlled trial. Aging Ment Health. 2003;7:195-9.

53. Hancock GA, Reynolds T, Woods B, Thornicroft G, Orrell M. The needs of older people with mental health problems according to the user, the carer , and the staff. Int J Geriatr Psychiatry. 2003;18:803-11.

54. Svendsboe EJ, Testad I, Terum T, Jörg A, Corbett A, Aarsland D, et al. Patterns of carer distress over time in mild dementia. Int J Geriatr Psychiatry. 2018;33:987-93.

55. Richters A, Melis RJ, van Exel NJ, Olde Rikkert MG, van der Marck MA. Perseverance time of informal caregivers for people with dementia: construct validity, responsiveness and predictive validity. Alzheimers Res Ther. 2017;9:26.

56. FFMS. Indicadores de educação em Portugal. PORDATA - Estatísticas, gráficos e indicadores de Municípios, Portugal e Europa. 2015. [consultado 2018 set 20]. http://www.pordata.pt/Municipios.

57. Bieber A, Stephan A, Verbeek H, Verhey F, Kerpershoek L, Wolfs C, et al. Access to community care for people with dementia and their informal carers: Case vignettes for a European comparison of structures and common pathways to formal care. Z Gerontol Geriatr. 2018;51:530-6.

58. Stephan A, Bieber A, Hopper L, Joyce R, Irving K, Zanetti O, et al. Barriers and facilitators to the access to formal dementia care: Findings of a focus group study with people with dementia, informal carers and health and social care professionals in eight European countries. BMC Geriatr. 2018;18:131

59. Broda A, Bieber A, Meyer G, Hopper L, Joyce R, Irving K, et al. Perspectives of policy and political decision makers on access to formal dementia care: expert interviews in eight European countries. BMC Health Serv Res. 2017;17:518.

60. Balsinha C, Gonçalves-Pereira M, Iliffe S, Freitas JA, Grave J. Health care delivery for older people with dementia in primary care. In: Mendonça-Lima C, Ivbijaro G, editors. Primary Care Mental Health for Older People. Berlin: Springer; 2019 [in press]. 